\title{
Automated Extraction of Cell Lineages and Spatial Migration Paths in Live $C$. elegans Embryos from 4D Fluorescence Microscopy Image Sequences
}

\author{
Ying Chen*, Gang Lin*, Omar Al-Kofahi*, William A. Mohler**, and Badrinath Roysam.* \\ * ECSE Department, Rensselaer Polytechnic Institute, Troy, NY 12180 \\ ** Genetics \& Developmental Biology, U. of Connecticut Health Center, Farmington, CT 06030
}

This paper presents fully-automatic methods for analyzing time-lapse 3D confocal image sequences of fluorescently tagged Caenorhabditis elegans worm embryos to extract the lineage, as well as three-dimensional (3D) spatial migration paths of cells. Prior work has focused on lineage extraction by laborious manual annotation, or automated methods that are limited to very early embryogenesis. They do not extract 3D cell migration paths during embryogenesis. This work overcomes both these limitations, and sets the stage for quantitative multi-dimensional studies of embryogenesis driven by multi-photon microscopy and fluorescent protein labeling.

Our method proceeds in four steps as illustrated in Fig. 1. First, time-lapse 3D two-photon microscopy of histone::GFP tagged $C$. elegans embryos is performed over long durations at 2minute intervals, with a high $3 \mathrm{D}$ resolution (6 pixels $/ \mu \mathrm{m} \times 63$ focal planes) at each time point [1]. Two-photon excitation at 900nm yielded high GFP signal from cell nuclei, low auto-fluorescence from unlabeled components, and tolerable photo-bleaching and photo-toxicity. Fig. 1 shows three sample frames from the 4D image data. Second, each of the cell nuclei and objects such as metaphase plates were segmented by a 3D watershed algorithm, as shown in Fig. 2 [2]. The resulting plot of cell counts over time was found to agree with published data. The segmentations were used to measure features such as cell location, volume, average intensity, intensity variability (chromatin texture), surface area, convexity, circularity, and eccentricity. Third, the segmented cell nuclei were tracked over time using these steps: i) quantify the similarity between nuclei identified at contiguous time-points based on measured features; ii) solve an $M \times N$ rectangular assignment problem [3] to determine optimal matches between $M$ nuclei detected at time $t$ and $N$ nuclei at $t+1$; iii) examine unassigned objects and refine assignments. Finally, the results of tracking were analyzed to identify cell divisions, cell lineage relationships at each division, and the 3-D migrations of each cell.

The results are rendered as color-coded lineage maps, and combined lineage and migration path maps (Fig. 3). Complete 4D visualization requires a computer. The automated analysis achieved a $68 \%$ accuracy for a lineage of $C$. elegans cleavage from 56 - 90 cell stages, covering a span of 140 170mins. post fertilization. Most errors occurred when sister nuclei migrated rapidly after division, indicating that improved temporal sampling may reduce errors. A user can edit the generated results to achieve significant automation, and benefit from a richer set of measurements.

\section{References}

1. Mohler, W.A. and A.B. Isaacson, in Imaging in Neuroscience and Development: A Laboratory Manual, Cold Spring Harbor Laboratory Press. pp. 119-124, 2005.

2. Lin, G., et al., Cytometry Part A, 63A:20-33, 2005.

3. Al-Kofahi, O., et al., Cell Cycle, 5: 3, pp: 327-335, 2006.

4. Supported by the NSF CenSSIS ERC (EEC-9986821) and NICHD (HD43156). 


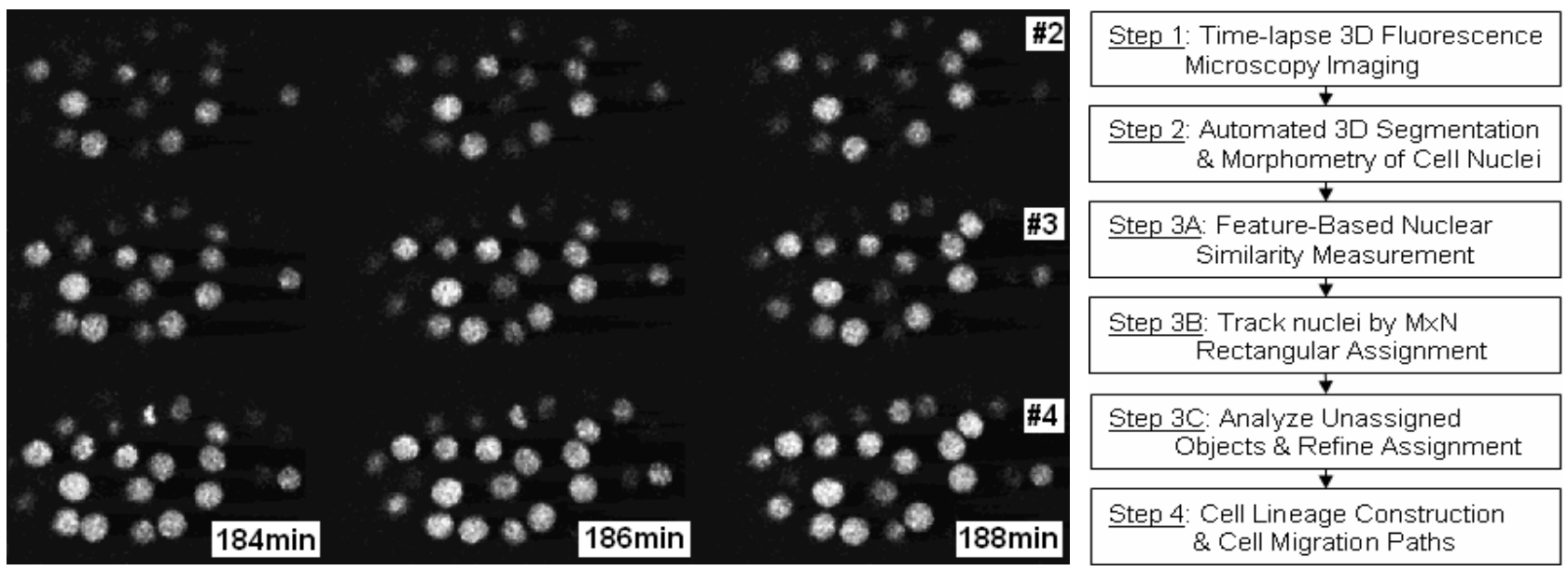

Fig. 1: (left) Sample 3D images of histone::GFP tagged C. elegans embryo at 3 time-points, and 3 focal planes. (Right) Main steps of the automated image analysis procedure to extract the lineage map for each cell, and 3D cell migration paths (shown in Fig. 3).
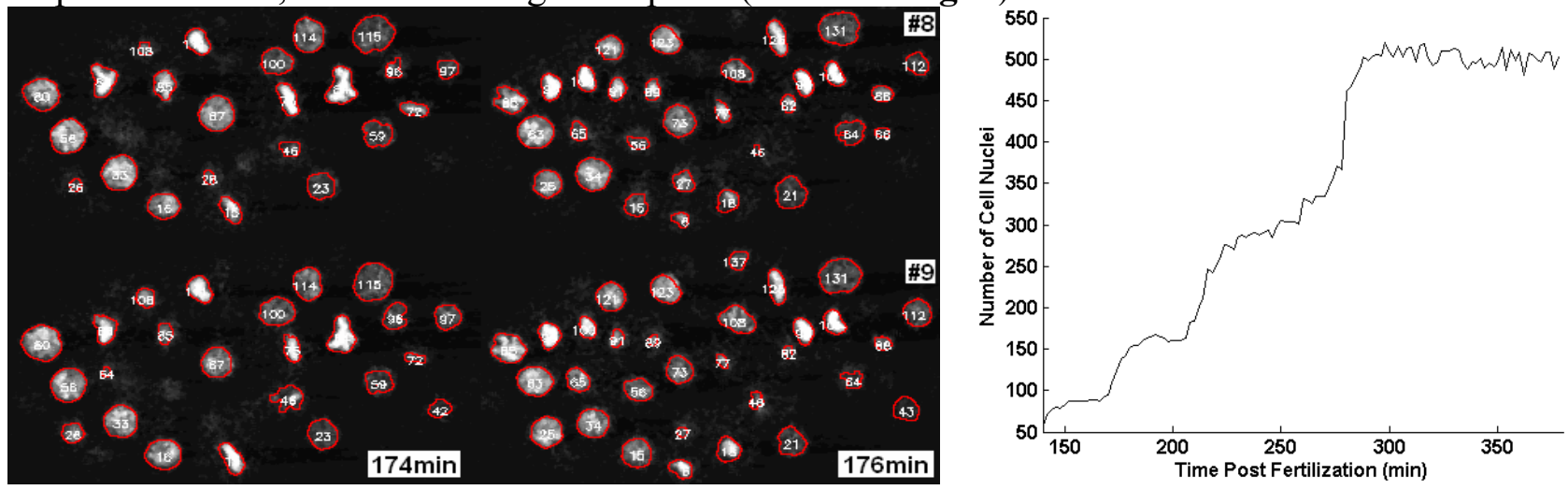

Fig. 2: (left) Sample automated nuclear segmentation results (Step 2) at 174 and 176 mins. at focal planes 8 and 9. Each object is numbered for reference. Elongated objects are metaphase plates. (right) Plot of cell counts from 140 - 380 minutes that concord with published data.

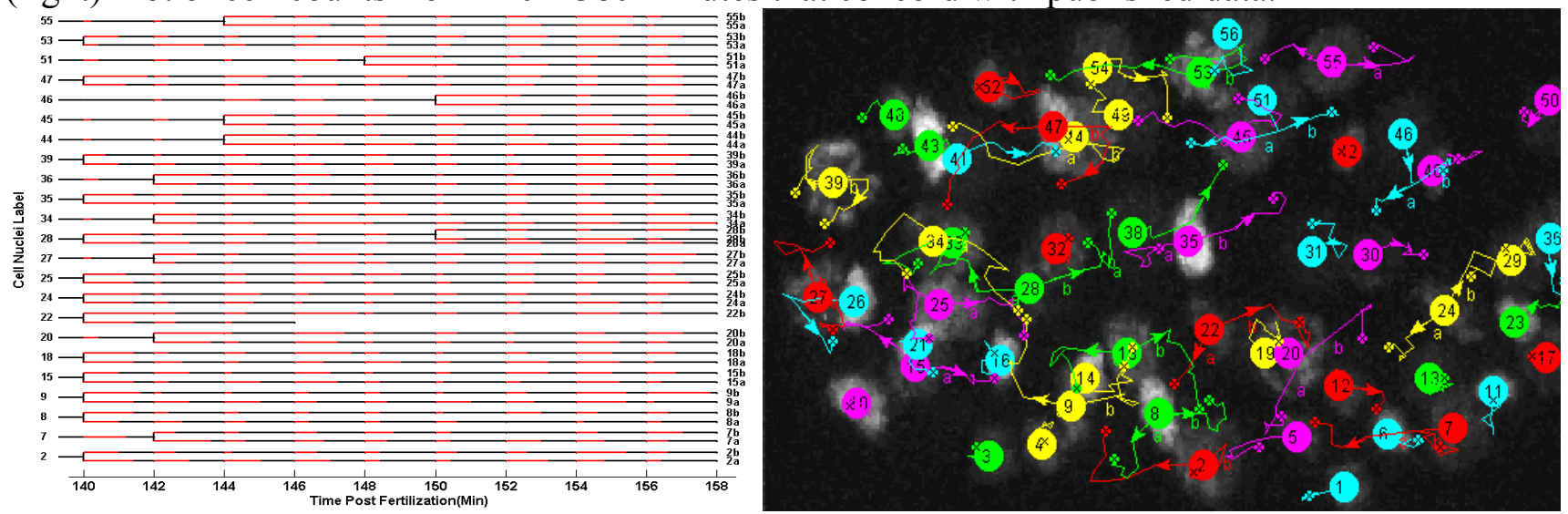

Fig. 3: Two methods to visualize results. (left) Partial color-coded lineage map for the first 18 minutes -red lines indicate relative migration distances. (right) Combined map for first 30 minutes showing initial cell locations as numbered circles, arrows indicating migration paths, and crosses indicating final locations of cells, overlaid on a maximum-value projection of the initial image. The complete results can be visualized three-dimensionally, and over time on a computer. 